\title{
Grenzen der Menschenrechte
}

\section{Territoriale Souveränität und die prekäre Rechtspersönlichkeit ${ }^{1}$ von Migrant*innen ${ }^{2}$}

\section{Borders of Human Rights}

\section{Territorial Sovereignty and the Precarious Personhood of Migrants}

\section{Ayten GÜNDOĞDU, New York}

Zusammenfasung: In diesem Artikel fokussiere ich auf die Kluft zwischen dem Begriff des „Menschen“, der den Menschenrechten zugrunde liegt, und dem Konzept der universellen Rechtspersönlichkeit, das ich im Hinblick auf die mediterranen Flüchtlingsbewegungen untersuche. In Anlehnung an Arendts berühmtes Diktum vom „Recht, Rechte zu haben“ analysiere ich die Spannung zwischen den souveränen Rechten von Staaten und den Rechtsansprüchen von Migrant*innen, was wiederum auf das hinweist, was ich als „Grenzen der Menschenrechte“ bezeichne: Sie beziehen sich auf die Begrenztheit der Menschenrechte angesichts der staatlichen Souveränität und sind in der Grenzkontrollpolitik der einzelnen Staaten und ihrer Grenzbehörden verkörpert. Wie ich unter Bezugnahme auf den Fall Hirsi vorschlage, sollte die Rechtspersönlichkeit von Migrant*innen jenseits territorialer Grenzen gedacht wer-

1 Auf Englisch „personhood“. Für die folgende Übersetzung wurde in Absprache mit der Autorin entschieden, jene Formulierungen von Arendt zu übernehmen, die sie in ihrem Buch Über die Revolution (1963) entwickelt und verwendet, und zwar: „Rechtspersönlichkeit“ (im Sinne von legal „personhood“) und ,juristische Person“ (als Gegenbegriff zu „natürlicher Mensch“).

2 Der Originalbeitrag „Borders of Human Rights: Territorial Sovereignty and the Precarious Personhood of Migrants" ist in Critical Perspectives on $\mathrm{Hu}$ man Rights, hrsg. von Birgit Schippers (Rowman \& Littlefield, 2019), 191212, erschienen. Übersetzung von Andreas Oberprantacher. 
den, während staatliche Funktionen, wie die Kontrolle der Staatsgrenzen, im Lichte der Menschenrechtsnormen überdacht werden müssen.

Schlagwörter: Menschenrechte; Rechtspersönlichkeit; Souveränität; Grenzregime; Flucht; Arendt; Mittelmeer

Abstract: In this article, I focus on the gap between the notion of the "the human" that underpins human rights and the concept of universal personhood, which I investigate with respect to the refugee movements across the Mediterranean. Drawing on Arendt's famous dictum of "the right to have rights", I analyze the tension between the sovereign rights of states and the rights claims of migrants that points up what I refer to as the "borders of human rights": they articulate the limitations of human rights in the face of state sovereignty and are embodied in the border-control policies implemented by individual states and their border agencies. As I suggest, drawing on the recent Hirsi case, the personhood of migrants should be reconsidered beyond the confines of territoriality, whereas state functions, such as the control of state borders, must be rethought in light of human rights norms.

Keywords: human rights; personhood; sovereignty; border regimes; refugees; Arendt; Mediterranean Sea

Das Mittelmeer ist zu einem Friedhof geworden: Wir haben mittlerweile die Nachrichten über „Tragödien von Migrant*innenbooten“ normalisiert, welche routinemäßig mit Fotos von gekenterten Booten und am Strand aufgereihten Leichensäcken präsentiert werden. Diese „Tragödien“ werden oft als Unfälle oder unglückliche Zwischenfälle behandelt, was es unmöglich macht, jemanden zur Verantwortung zu ziehen. Doch wer mit der Grenzpolitik der europäischen Staaten seit Ende der 1990er Jahre etwas vertraut ist, wird kaum übersehen, dass es genau diese Politik ist, die den grenzüberschreitenden Personenverkehr kriminalisiert, das Asylrecht erheblich eingeschränkt und Migrant*innen zu immer gefährlicheren Reisen gezwungen hat. Die vorherrschenden Narrative, die als Reaktion auf das gegenwärtig massive Ausmaß erzwungener Deplatzierung entworfen wurden und auf Begriffe wie „Krise“ und „Sicherheit“ fokussieren, machen es uns jedoch unmöglich, diese Todesfälle als „Morde“ anzuerkennen. Die toten Migrant*innen werden zu den heutigen homines sacri, um Giorgio Agambens Terminus zu verwenden: Sie werden zu nackten Leben gemacht, die ungestraft getötet werden können und für deren Tod es nicht einmal ein Gedenken gibt (Agamben 1998, 81-86). 
Dieses Problem betrifft uns angesichts dieser so genannten Unfälle sehr stark. Es gibt keine etablierten Verfahren, um die Toten zu identifizieren, ihre Familien zu informieren und sie mit angemessenen Ritualen zu bestatten. Die Leichen von Migrant*innen können oft nicht geborgen werden und wenn sie es doch werden, bleiben sie undokumentiert und ihre Familien werden nicht einmal benachrichtigt. In einer Zeit, die von Statistiken besessen ist, führt die Europäische Union keine offizielle Zählung der Toten oder Vermissten, was unverkennbar für die institutionelle Normalisierung der Grenzpolitik spricht, die Migrant*innen im Leben und im Tod entmenschlicht (siehe Robins, Kovras und Vallinatou 2014, 5).3 Das Missing Migrants Project, das von der Internationalen Organisation für Migration als Reaktion auf den Tod von mindestens 368 Migrant*innen in der Nähe der italienischen Insel Lampedusa im Oktober 2013 ins Leben gerufen wurde, hat im Jahr 2016 weltweit 7927 Migrant*innen als tot oder vermisst erfasst und berichtet, dass 5143 dieser Todesfälle im Mittelmeer zu verzeichnen waren. ${ }^{4}$ Selbst bei diesen konservativen Schätzungen sprechen wir von nicht weniger als einem Massaker.

Das Mittelmeer als Friedhof von Migrant*innen macht eine Erörterung der Grenzen der Menschenrechte dringend erforderlich. Genauer gesagt verlangt es sorgfältige Aufmerksamkeit für die Einschränkungen dieser Rechte, wenn es darum geht, die tödliche Grenzkontrollpolitik infrage zu stellen, die im Namen der territorialen Souveränität gerechtfertigt wird. Besonders dringlich ist in diesem Zusammenhang eine kritische Auseinander-

$3 \quad \mathrm{Zu}$ den Todesfällen von Migrant*innen siehe u.a. Grant 2011. Zu den Gründen, die der Schwierigkeit der Schätzung von Todesfällen zugrunde liegen, siehe Frontex 2016. Angesichts des offiziellen Schweigens und der Vernachlässigung von offizieller Seite gibt es nun verschiedene Bemühungen an der Basis, die Toten zu identifizieren und richtig zu bestatten, ihres Lebens zu gedenken und ihre Angehörigen zu informieren; siehe z. B. Rygiel 2014. Eine starke Ausnahme vom offiziellen Schweigen und der Vernachlässigung auf internationaler Ebene ist der jüngste Bericht von Agnes Callamard, der Sonderberichterstatterin des Menschenrechtsrates zu extralegalen, summarischen oder willkürlichen Hinrichtungen, siehe UN-Generalversammlung 2017. Für eine aufschlussreiche Analyse des Berichts siehe Kalpouzos und Mann 2017.

4 Vergleiche diese Zahlen mit den im Jahr 2015 gemeldeten Zahlen: Weltweit wurden 6259 Migrant*innen als tot oder vermisst gemeldet und 3784 im Mittelmeerraum. Internationale Organisation für Migration, „Missing Migrants Project“, abgerufen am 9. Juni 2017, http://missingmigrants.iom.int/latest-global-figures. 
setzung mit der recht eigentümlichen Formulierung „die menschliche Person", welche in Menschenrechtsdokumenten wiederholt beschworen wird. So bekräftigt die Allgemeine Erklärung der Menschenrechte „die Würde und den Wert der menschlichen Person" und im Internationalen Pakt über bürgerliche und politische Rechte heißt es, dass sich die Menschenrechte „aus der dem Menschen innewohnenden Würde herleiten“. Durch die Verbindung zweier Begriffe, die während des größten Teils der Geschichte getrennt blieben (d.h. „Mensch“ und „Person“), verkünden diese Dokumente, dass jedes menschliche Wesen als eine vor dem Gesetz gleichberechtigte Person anerkannt werden soll. Die Todesfälle von Migrant*innen im Mittelmeerraum und darüber hinaus werfen zwei miteinander verbundene Fragen in Bezug auf diese etablierte Norm der universellen Rechtspersönlichkeit auf: Erstens, inwieweit erlaubt es der bestehende Menschenrechtsrahmen den Migrant*innen, als vor dem Gesetz, im Leben und im Tod gleichberechtigte Personen aufzutreten? Zweitens, in welchem Ausmaß und auf welche Weise kann der Menschenrechtsrahmen für die Zwecke der Kritik an der Grenzpolitik mobilisiert werden, die Migrant*innen zu Nichtpersonen im Leben und im Tod macht?

Im Folgenden gehe ich auf diese Fragen ein, indem ich mich auf Hannah Arendts Diskussion über Staatenlosigkeit beziehe, insbesondere auf ihre Bemerkungen zum Verlust der Rechtspersönlichkeit als ein bestimmendes Merkmal des Zustands der Rechtlosigkeit, den Staatenlose erleiden. Ich werde dies tun, indem ich einen Fall des Europäischen Gerichtshofs für Menschenrechte aus dem Jahr 2012 untersuche: Hirsi Jamaa and Others v. Italy. Europäische Staaten haben zunehmend auf das Abfangen auf hoher See zurückgegriffen, um Migrant*innen zu stoppen, bevor sie Land erreichen konnten, und um sich den Verpflichtungen zu entziehen, die sich durch die internationalen Menschenrechte und das humanitäre Recht ergeben. Der Fall Hirsi betrifft direkt die Verfügbarkeit von Grundrechten für Migrant*innen, die auf hoher See abgefangen werden. Das Gericht entschied zugunsten der Migrant*innen und argumentierte, dass Italien ihre Rechte verletzt habe, indem es ihre Ankunft blockiert und ihnen die Möglichkeit verweigert habe, Asyl zu beantragen, und sie schließlich nach Libyen zurückgeschickt habe. Verschiedene Anwaltsgruppen begrüßten das Urteil als eine unbestreitbare Bestätigung der extraterritorialen Anwendung von Menschenrechtsnormen und als einen kräftigen Schlag gegen die Bemühungen jener Staaten, welche von den Migrant*innen angesteuert werden, die hohe See in eine gesetzlose Zone zu verwandeln. 
Indem ich einen kritischen Rahmen Arendt'scher Prägung entwickle, stelle ich Fragen zu dieser Schlussfolgerung und erörtere das Hirsi-Urteil als beispielhafte Veranschaulichung für die anhaltenden Aporien bezgl. der Menschenrechte, insbesondere die herausfordernden Probleme, die sich aus der Verflechtung der Prinzipien der universellen Rechtspersönlichkeit und der territorialen Souveränität in diesem Rahmen ergeben. Diese Aporien sind umso greifbarer geworden, seit Staaten sich jenseits ihrer territorialen Jurisdiktion mit Praktiken der Migrationskontrolle, einschließlich des Abfangens auf See, befassen. Eine solche Politik stellt das herkömmliche Bild der Grenze als feste, statische Grenze infrage und führt uns zu „schwankenden“ Grenzen, um Étienne Balibar zu zitieren, welche sich beim Management von Bevölkerungen ständig verschieben und verbreitern (Balibar 2002, 92). ${ }^{5}$ In einem internationalen System, das die Rechte nach wie vor an das Territorialitätsprinzip bindet, reduzieren diese neuartigen Grenzpraktiken die meisten Migrant*innen auf den Status einer prekären Rechtspersönlichkeit. Diese zeitgenössischen Probleme haben einige kritische Theoretiker*innen wie Roberto Esposito zur weitreichenden Schlussfolgerung geführt, dass Menschenrechte, die auf das Konzept der juristischen Person fokussieren, notwendigerweise verschiedene Formen gewaltsamer Ausgrenzung mit sich bringen und rechtfertigen (siehe Esposito 2012a und 2012b). Um diese Schlussfolgerung infrage zu stellen, endet das Kapitel mit einer Diskussion der gesonderten Stellungnahme von Richter Pinto de Albuquerque im Fall Hirsi. Diese Stellungnahme kann als Ausgangspunkt dienen, um einige der Beschränkungen in der Argumentation des Gerichtshofs zu identifizieren, die Idee zu problematisieren, dass die territoriale Souveränität den Staaten ein absolutes Recht gibt, ihre Grenzen zu kontrollieren, und die Menschenrechte im Sinne dessen, was Arendt als „ein Recht, Rechte zu haben“ bezeichnete, neu zu überdenken.

$5 \quad$ Siehe auch Vaughan-Williams 2009, Kapitel 1. Die Frage, ob Grenzen jemals feste, statische Grenzen waren, ist umstritten; siehe Mezzadra und Neilson 2013; Nail 2016. 


\section{Staatenlosigkeit als Lackmustest für die Menschenrechte ${ }^{6}$}

Hannah Arendts Diskussion der Staatenlosigkeit in Elemente und Ursprünge totaler Herrschaft liefert entscheidende Ressourcen zur Diskussion dieser Probleme (Arendt 1968, 267-302). Als sie über das massive Ausmaß des Problems der Staatenlosigkeit in Europa in der ersten Hälfte des 20. Jahrhunderts schrieb, beobachtete Arendt ein verblüffendes Paradoxon: Diejenigen, die in ihrer nackten Menschlichkeit erschienen, d.h. aller sozialen und politischen Attribute beraubt wurden, hatten keinen Zugang zu den Rechten, die sie infolge ihrer menschlichen Geburt angeblich hätten besitzen sollen; der Verlust der Staatsbürgerschaft führte zum Verlust der Menschenrechte: "If a human being loses his political status, he should, according to the implications of the inborn and inalienable rights of man, come under exactly the situation for which the declarations of such general rights provided. Actually the opposite is the case. It seems that a man who is nothing but a man has lost the very qualities which make it possible for other people to treat him as a fellow-man." (Arendt 1968, 300)

Arendt nahm dieses Paradoxon als Symptom für „die Aporien der Menschenrechte" und befasste sich vor dem Hintergrund des Problems der Staatenlosigkeit mit den Annahmen, die der Erklärung der Menschen- und Bürgerrechte von 1789 zugrunde lagen. Man ging davon aus, dass die „Rechte des Menschen“ natürliche Rechte seien, die unabhängig von der Mitgliedschaft in einer politischen Gemeinschaft gegeben seien; man ging davon aus, dass sie dem „Menschen“ gehörten, verstanden als „an ,abstract“ human being“, das „carried his dignity within himself without reference to some larger encompassing order“ (Arendt 1968, 291). Aber wie aus dem Titel der Erklärung von 1789 hervorgeht, wurde dieser „Mensch“ auch als „Bürger“ oder als Mitglied eines Nationalstaates identifiziert (Arendt 1968, 291). Die Probleme mit dieser Identifizierung wurden zu Beginn des 20. Jahrhunderts in gefährlicher Weise spürbar, als rassische und ethnische Kategorisierungen von Bevölkerungen zu einer massenhaften Denaturalisierung und Entnationalisierung führten, die Millionen ihrer Bürgerrechte beraubte und sie in einem Zustand der Rechtlosigkeit zurückließ (Arendt 1968, 278-280).

Arendts Erörterung der Staatenlosigkeit bietet eine facettenreiche Darstellung der Rechtlosigkeit der Staatenlosen. Rechtlich gesehen besteht das Problem im Verlust der Rechtspersönlichkeit; ohne jede formale Aner-

6 Dieser Abschnitt stützt sich auf meine Analyse von Arendts Argumenten, die ich an anderer Stelle durchführe, siehe Gündoğdu 2015. 
kennung durch nationale und internationale Gesetze können die Staatenlosen nicht als Rechtssubjekte vor dem Gesetz stehen. Infolgedessen sind sie der Gesetzlosigkeit und „willkürlichen Polizeireglements“ ausgeliefert, unfähig, Grundbedürfnisse wie Nahrung und Wohnen als Rechte einzufordern, und immer „liable to jail sentences without ever committing a crime“ (Arendt 1968, 286). Darüber hinaus bedeutet „Rechtlosigkeit“ die Verstoßung aus einem politischen Rahmen, der die eigenen Rechte wirksam schützen könnte - nicht nur den „Verlust des staatlichen Schutzes“, sondern auch den Verlust einer politischen Gemeinschaft, in der den Worten und Taten des Einzelnen Rechnung getragen wird (Arendt 1968, 294, 296). Angesichts dieser rechtlichen und politischen Probleme wird es für Staatenlose schwierig, als Menschen in ihrer unverwechselbaren Individualität anerkannt zu werden; sie werden oft auf „mere existence“ als „some specimen of an animal species, called man“ (Arendt 1968, 301-302) reduziert und als mittellose Körper wahrgenommen, die gefüttert, gekleidet und behütet werden müssen. Diese „expulsion from humanity“ wird durch die Einsperrung der Staatenlosen in Lagern verkörpert, die sie physisch vom Rest der Welt absondern (Arendt 1968, 297).7

Die Betonung, die Arendt auf die Schwierigkeiten der Staatenlosen legt, ihre Worte und Taten in der Gesellschaft anderer Menschen relevant $\mathrm{zu}$ machen, ist für diejenigen, die mit ihrer politischen Theorie vertraut sind, nicht allzu überraschend. Immerhin ist sie bekannt für ihr Bekenntnis zum Handeln als Fähigkeit, Neuanfänge zu initiieren, für ihre Darstellung der Freiheit und für die entscheidende Rolle, die sie dem Sprechen bei der fortschreitenden Gleichstellung der Menschen beimisst (siehe z. B. Arendt 1998, 175-181; Arendt 2005, 113, 118). Aber die Aufmerksamkeit, die sie dem Problem der Rechtspersönlichkeit in ihrer Darstellung der Staatenlosigkeit widmet, ist recht auffallend, vor allem weil die rechtlichen Dimensionen ihres Denkens wenig wissenschaftliche Beachtung gefunden haben. ${ }^{8}$ Angesichts der gegenwärtigen Grenzpolitik, die es Migrant*innen zunehmend erschwert, als Personen vor dem Gesetz aufzutreten, verdienen Arendts Überlegungen zu den rechtlichen Problemen der Staatenlosen eine nähere Betrachtung.

$7 \quad$ Für eine Arendt'sche Analyse der Schäden, die durch Langzeitlager verursacht werden, siehe Gündoğdu 2015; Parekh 2017.

$8 \quad \mathrm{Zu}$ Arendts Rechtsverständnis siehe u.a. Goldoni und McCorkindale 2012; Maxwell 2012; Volk 2015; Waldron 2000. 
Im Zentrum dieser Überlegungen steht ein Vergleich, den Arendt zwischen „Kriminellen“ und „Staatenlosen“ zieht. Beide Figuren werden im Rechtssystem als Ausnahmen von der Norm angesehen, da ihnen Grundrechte, einschließlich des Rechts auf Freizügigkeit, verweigert werden. Doch in den Augen des Gesetzes, so Arendts Darstellung, befinden sich diejenigen, die eines Verbrechens angeklagt sind, in einer besseren Position als die Staatenlosen. Es handelt sich um „a status of recognized exception“ innerhalb „the general law“, während Staatenlosigkeit „an anomaly for whom the general law [does] not provide“ (Arendt 1968, 287, 286) ist. Diese Behauptung sollte vor dem Hintergrund der Tatsache bedacht werden, dass der Status und die Rechte der Staatenlosen zu jener Zeit, als Arendt über das Problem schrieb, in den innerstaatlichen und internationalen Gesetzen nicht formell anerkannt worden sind; daher besteht sie darauf, dass „[t]heir plight is not that they are not equal before the law, but that no law exists for them" (Arendt 1968, 295-296). Ohne eine solche Anerkennung können die Staatenlosen einer „form of lawlessness, organized by the police“ unterworfen werden; ihre Grundbedürfnisse werden in einem kapriziösen Rahmen der Barmherzigkeit befriedigt, jedoch nicht als Rechte garantiert, auf die sie Anspruch hätten (Arendt 1968, 288, 296).

Die Figur des „Kriminellen“ erlaubt es Arendt, die ausgeprägte rechtliche Zwangslage von „Staatenlosen“ hervorzuheben: Während die Strafe innerhalb des Strafrechtssystems angeblich aus der Tat der Übertretung abgeleitet wird und in einem angemessenen Verhältnis zu dieser stehen sollte, ist das, was die Staatenlosen erleiden, völlig willkürlich in dem Sinne, dass es keine „relation whatsoever to what they do, did, or may do“ (Arendt 1968, 296, siehe auch 447) hat. Unter diesen Bedingungen, so argumentiert Arendt provokativ, kann die Begehung eines Verbrechens für die Staatenlosen die einzige Möglichkeit sein, ihren Status zu verbessern; nur dann würden die Staatenlosen als Person vor dem Gesetz anerkannt und ihnen Grundrechte wie das Recht auf ein Gerichtsverfahren, rechtliche Vertretung und Berufung gewährt werden: „He is no longer the scum of the earth but important enough to be informed of all the details of the law under which he will be tried. He has become a respectable person.“ (Arendt 1968, 286-287)

Arendts scharfe Unterscheidung zwischen „Staatenlosen“ und „Kriminellen“ ist heutzutage schwer aufrechtzuerhalten. Der Aufstieg eines Masseninhaftierungssystems, insbesondere die unverhältnismäßig hohen Inhaftierungsraten von Minderheiten in den Vereinigten Staaten, und die zunehmende Kriminalisierung von Migrant*innen in Europa und Nord- 
amerika haben diese Unterscheidung in vielerlei Hinsicht verwischt. Das Strafrechtssystem nimmt zunehmend die Merkmale einer polizeilichen Willkürherrschaft an, die mit dem System der Einwanderungshaftanstalten verbunden ist, insbesondere infolge der Privatisierung der Gefängnisse. Der grundlegende Schutz gemäß rechtsstaatlichen Prinzipien ist für eine wachsende Zahl von Menschen, die de facto staatenlos geworden sind, unabhängig von ihrem Staatsangehörigkeitsstatus, zudem kaum noch gegeben. ${ }^{9}$

Diese Unschärfe anzuerkennen, entkräftet jedoch nicht Arendts Argument, wie bedeutsam die Anerkennung als Person vor dem Gesetz ist. In der Tat kann ihre Arbeit entscheidende Ressourcen für die kritische Analyse von Praktiken des Zunichtemachens von Rechtspersönlichkeit liefern - unabhängig davon, ob sie im Strafrechtssystem, im Grenzkontrollsystem oder an deren Schnittpunkten angesiedelt sind -, durch die eine ständig wachsende Zahl „unerwünschter Elemente“ geschaffen wurde, die in Zonen der Gesetzlosigkeit eingeschlossen, von der Welt der Lebenden isoliert und zu einer bestimmten Form des zivilen und sozialen Todes verurteilt wurden (Arendt 1968, 440).

Dieser letzte Punkt wirft eine neue Frage auf: Würden wir angesichts all der Entwicklungen auf dem Gebiet der Menschenrechte seit dem Zeitpunkt, an dem Arendt ihre Analyse der Staatenlosigkeit abgeschlossen hat, nicht erwarten, dass die legale Dimension der Rechtlosigkeit verschwindet? Besonders wichtig ist in diesem Zusammenhang die Einführung der „menschlichen Person“ als Subjekt von Rechten in mehreren Menschenrechtsdokumenten - ein Schritt, der sich erheblich von der Identifizierung von „Mensch und Bürger“ im Rechtsdiskurs des 18. Jahrhunderts unterscheidet. ${ }^{10}$ Es ist durchaus verlockend anzunehmen, dass diese Verschiebung die Aporien, die im Mittelpunkt von Arendts Analyse stehen, gelöst habe. Während Staatsbürgerschaft im Laufe ihrer Geschichte verschiedene Gruppen ausgeschlossen hat (z. B. Frauen, erwerbstätige Arme, Einwander*innen), scheint die Rechtspersönlichkeit als universalistische Kategorie das Versprechen zu halten, solche Ausschlüsse zu überwinden und alle Men-

9 Für einen erhellenden Bericht, der untersucht, wie kriminalisierten People of Color Rechtspersönlichkeit verweigert wird, siehe Cacho 2012.

Zum Übergang von der Staatsbürgerschaft zur Persönlichkeit als Grundlage für den Anspruch auf Rechte innerhalb eines menschenrechtlichen Rahmens siehe u. a. Benhabib 2004; Cohen 1999; Jacobson 1996; Sassen 2002; Soysal 1994. 
schen unter ihren Schutzschirm zu nehmen. Darüber hinaus wird im Gegensatz zur Staatsbürgerschaft, die verloren gehen oder weggenommen werden kann, die Rechtspersönlichkeit oft als ein besonderer menschlicher Status verstanden, der auf einem inhärenten und daher unantastbaren Merkmal wie Heiligkeit, Würde, Vernunft oder Autonomie beruht. ${ }^{11}$

Wie verschiedene Forscher*innen, die sich mit dem Thema der Rechtspersönlichkeit befassen, allerdings hervorgehoben haben, behindern diese weit verbreiteten Annahmen das Verständnis jener Praktiken, die kontinuierlich durch Gesetze Personen machen oder zunichtemachen, bestimmte Subjekte von der rechtlichen Gleichstellung ausschließen und eine Kluft zwischen der bloßen Tatsache der Menschlichkeit und der Rechtspersönlichkeit offenlegen (siehe u.a. Cacho 2012; Dayan 2011; Esposito 2012a, 2012b und 2015; Naffine 2009). Arendts politische Theorie bietet entscheidende Ressourcen für die Formulierung eines kritischen Rahmens, der sich kritisch auf die zeitgenössischen Manifestationen dieser Kluft beziehen ließe. Diese Ressourcen finden sich nicht nur in ihrer Schilderung der Staatenlosigkeit, sondern auch in ihren Ausführungen über die juristische Person im römischen Recht.

Arendt bietet ein einzigartiges Verständnis von Rechtspersönlichkeit, indem sie sich auf deren etymologische Ursprünge im lateinischen Begriff persona besinnt, welcher „the mask ancient actors used to wear in a play“ (Arendt 1990, 106; siehe auch Arendt 2003a, 12; Arendt 2003b, 12-13) bezeichnet. Sie unterstreicht, dass es die Römer waren, die persona zum ersten Mal in einem metaphorischen Sinne benutzten, um eine rechtliche Unterscheidung zwischen Privatpersonen und Bürgern zu treffen. Die Römer betrachteten die juristische Person als einen künstlichen, vom Recht geschaffenen Status und nicht als ein inhärentes Attribut, das allen Menschen in ihrem natürlichen Zustand gemeinsam wäre: „The point was that ,it is not the natural ego which enters court of law. It is a rightanddutybearing person, created by the law, which appears before the law'" (Arendt 1990, 107; siehe auch Arendt 2003a, 12-13) Indem sie einen etymologisch fragwürdigen Schritt macht, teilt Arendt ihren Leser*innen auch mit, dass sich persona von per-sonare herleitet, was „sound through"12 bedeutet. Zusammen mit dieser Etymologie schlägt sie vor, dass die Maske „a broad opening at

11 Für eine Kritik dieser Annahmen siehe z. B. Bosniak 2010 und 2011.

12 Arendt ist sich der Schwierigkeiten bei der Rückverfolgung von persona zu per-sonare bewusst, siehe Arendt 1990, 293n. Zur umstrittenen Etymologie der persona siehe z. B. Trendelenburg 1910, 6-8. 
the place of the mouth through which the voice of the actor could sound through" (Arendt 2003a, 12) habe.

Arendts eigentümliche Etymologie der juristischen Person ist vor allem aus zwei Gründen wichtig: Indem sie die Rechtspersönlichkeit als Artefakt und nicht als intrinsisches menschliches Attribut betrachtet, unterstreicht sie erstens, dass es keine notwendige Überschneidung zwischen „dem Menschlichen“ und „der Person“ gibt. Mit diesem Schritt stellt Arendt „die Metaphysik der Person“ infrage, um John Deweys Ausdruck zu verwenden, welcher Rechtspersönlichkeit einem wirklichen oder wesentlichen menschlichen Wesen zuordnet, das vermutlich Rechtsbeziehungen vorausgeht und infolge eines angeblich inhärenten Merkmals wie Heiligkeit oder Würde eindeutig zur juristischen Person taugt. Arendts Misstrauen gegenüber einer solchen Metaphysik zeigt sich in ihrem Bericht zur Rechtlosigkeit der Staatenlosen, der den Zusammenbruch einer solchen Metaphysik ankündigt: „The conception of human rights, based upon the assumed existence of a human being as such, broke down at the very moment when those who professed to believe in it were for the first time confronted with people who had indeed lost all other qualities and specific relationships - except that they were still human. The world found nothing sacred in the abstract nakedness of being human." (Arendt 1968, 299)

Arendts kritische Darstellung der Staatenlosigkeit führt uns zu Subjekten, die, der Maske der juristischen Person entkleidet, „humanly unrecognizable“ oder „less than human without entitlement to rights" geworden sind, wie Judith Butler es formuliert (Butler 2004, 98). Diese Darstellung unterstreicht die Notwendigkeit, das Konzept der "menschlichen Person“ zu hinterfragen, das im zeitgenössischen Menschenrechtsdiskurs häufig beschworen wird, und deutet auf die Möglichkeit einer Kluft zwischen diesen beiden miteinander verbundenen Begriffen hin - d.h., nicht jeder Mensch wird vor dem Gesetz automatisch als Person anerkannt und selbst wenn man diese formale Anerkennung hat, können verschiedene Praktiken des Zunichtemachens von Rechtspersönlichkeit diese so weit verringern, dass manche Menschen zu Semi- oder sogar Nichtpersonen werden.

Zweitens verdeutlicht Arendts Etymologie der juristischen Person als per-sonare auch, warum das Recht, vor dem Gesetz zu stehen, politisch bedeutsam ist, insbesondere wenn man es mit Blick auf die liminalen Gestalten, die an den Grenzen der Rechtspersönlichkeit wohnen, einschließlich der Staatenlosen, neu überdenkt. Um die Etymologie in Erinnerung zu rufen, verbirgt die künstliche Maske der persona nicht nur das Gesicht der Schauspielerin, 
sondern lässt auch die Stimme „durchklingen“. Die verbergende Funktion der Maske lässt vermuten, dass die juristische Person eine Form der Schutzverkleidung bietet, als wolle sie vor der willkürlichen Staatsmacht schützen. ${ }^{13}$ Darüber hinaus drängt uns Arendts phänomenologisches Verständnis der Welt dazu, dieses Verbergen als eine Form der Erscheinung oder Selbstdarstellung zu verstehen; ${ }^{14}$ die künstliche Maske der persona ist wichtig, um die immer täuschende und doch so wichtige Möglichkeit zu eröffnen, als Gleiche unter Gleichen auf der juristischen Bühne mitzuerscheinen. Schließlich ist auch zu beachten, dass die Etymologie Arendts der Maske eine auditive Dimension zuweist; die Öffnung der Maske ermöglicht es, die „sonore Emission“ der Schauspielerin als Anspruch auf Rechte und nicht als unverständliches Geräusch zu hören. ${ }^{15}$ Arendts Darstellung der persona legt nahe, dass die Anerkennung der Rechtspersönlichkeit nicht nur eine juristische Frage ist, sondern auch eine politische Angelegenheit, die direkt mit der Frage verbunden ist, wer in einer politischen Gemeinschaft sichtbar und hörbar ist.

Diese beiden Punkte sind wichtig, um zu verstehen, was Arendt mit einigen zeitgenössischen Kritiker*innen der Menschenrechte, der juristischen Person und des Rechts teilt und wo sie von ihnen abweicht. Roberto Esposito verdient in diesem Zusammenhang vor allem deshalb Aufmerksamkeit, weil seine Kritik der juristischen Person und der Menschenrechte sich wesentlich auf Arendts Werk bezieht. ${ }^{16}$ Esposito applaudiert Arendt als „[t]he only thinker who has come close to tackling the question“ (Esposi-

13 Arendts Aufmerksamkeit für die Verwundbarkeit der Staatenlosen gegenüber willkürlicher Polizeiherrschaft scheint sie in die Nähe neorepublikanischer Theoretiker*innen (z. B. Pettit 1997) zu bringen, die Freiheit als Nichtherrschaft oder Unabhängigkeit von willkürlicher Macht verstehen. Doch Arendt bleibt nicht einfach dabei stehen, lenkt ihr phänomenologischer Ansatz doch die Aufmerksamkeit darauf, wie aus juristischen Fiktionen wie der Rechtspersönlichkeit neue Formen des öffentlichen Erscheinens entstehen. Freiheit entsteht aus Räumen der Erscheinung, indem wir gemeinsam handeln und Worte und Taten teilen, so Arendt; sie ist mit Nichtherrschaft verbunden, aber nicht auf sie reduzierbar.

14 Für eine Studie, welche die immer zweideutige Funktion von Masken als enthüllende und verbergende Artefakte hervorhebt, siehe Belting 2017.

15 Diese Formulierung stützt sich auf Rancière 1999, 22-23.

16 Ein weiterer Denker, der in dieser Hinsicht wichtig ist, ist Giorgio Agamben. Ich konzentriere mich auf das Werk von Esposito, weil es das Konzept der „juristischen Person“ hinterfragt. 
to 2012a, 68) im Zusammenhang mit dem Versagen der Menschenrechte, menschliches Leben zu schützen, und charakterisiert sie als eine Denkerin, die Gesetz und Rechte als notwendigerweise ausschließend betrachtet. Der Zustand der Staatenlosen, so Espositos Lesart von Arendt, offenbart „the structurally exclusive mechanism of rights“ (Esposito 2012a, 69). Der menschenrechtliche Rahmen versäumt es unweigerlich, die Rechte auf alle Menschen auszudehnen und das menschliche Leben in all seinen Dimensionen zu schützen, so seine Schlussfolgerung, gerade weil er die Rechte an die juristische Person bindet (siehe Esposito 2012a, 5). Diese Folgerung beruht auf Espositos Behauptung, dass die juristische Person immer schon als ein Mechanismus des gewaltsamen Ausschlusses oder als ein dispositif gewirkt hat, um jenen Begriff zu verwenden, den er von Michel Foucault entlehnt hat, indem Trennungen zwischen den Menschen und innerhalb jedes Menschen installiert wurden. Das römische Recht spielt eine entscheidende Rolle in dieser kritischen Darstellung, da es die Trennung zwischen persona und homo bzw. die juristische Fiktion der Rechtspersönlichkeit und der singulären, körperlichen menschlichen Existenz einführt. Für Esposito verkapselt die Kluft zwischen Gesicht und Maske, illustriert durch die römische persona, die Diskrepanz zwischen menschlichem Leben und seiner rechtlichen Darstellung. Die Menschenrechte überwinden diese Kluft nicht, sondern reproduzieren sie gerade deshalb, weil sie die juristische Person als Grundlage der Rechte nehmen.

In mancher Hinsicht folgt die Analyse von Esposito der Analyse der Staatenlosigkeit von Arendt. Immerhin weigert sich Arendt, die Rechtlosigkeit der Staatenlosen als einen unglücklichen Unfall zu sehen, und betrachtet diese, wie bereits erwähnt, stattdessen als ein Symptom der tief verwurzelten Aporien der Menschenrechtsnormen innerhalb eines nationalstaatlichen Systems. Darüber hinaus weist ihr Einsatz des römischen Rechts auf eine Kluft zwischen der bloßen Tatsache des Menschseins und dem rechtlichen Status der juristischen Person hin, indem sie die Aufmerksamkeit auf jene Figuren lenkt, die auf das bloße Leben reduziert und aus dem Bereich des Gesetzes gedrängt werden.

Diese Diagnosen führen sie aber auch zu einem ganz anderen kritischen Zugang zu Menschenrechten, juristische Person und Recht. Ihre Analyse der Staatenlosigkeit führt sie nicht zu Espositos Schlussfolgerung, dass es „an anomaly in the juridical procedure as such“ gebe oder dass „the human being as such is precisely what law excludes from within its borders“ (Esposito 2012a, 69). Die Aporien der Menschenrechte sind für Arendt keine 
Sackgassen, die ein „essential failure“ (Esposito 2012a, 5) symptomatisieren. Sie sind herausfordernde politische und ethische Dilemmata, die den modernen Rechtsdiskurs seit dem 18. Jahrhundert charakterisieren. Es besteht aber immerhin die Möglichkeit, sie auf eine Weise zu umschiffen, welche die Möglichkeiten eröffnet, gleiche Rechte für alle einzufordern. Dies ist vielleicht der Grund, warum sich Arendts Kritik nicht ganz von den Menschenrechten abwendet, sondern stattdessen vorschlägt, sie als „das Recht, Rechte zu haben" neu zu denken, was das Recht einschließt, als Person vor dem Gesetz zu stehen, das Recht, in einer politischen Gemeinschaft zu leben, in der die eigenen Handlungen und Meinungen berücksichtigt werden, und das Recht, der Menschheit anzugehören (siehe Arendt 1968, 296-298).

Arendts Kritik an den Menschenrechten spiegelt auch wider, wie sich ihr Rechtsverständnis deutlich von dem zeitgenössischer Kritiker*innen wie Esposito unterscheidet. In „Guests from No-Man's-Land“, einem kurzen Artikel, den sie 1944 für die deutsch-jüdische Zeitung Aufbau schrieb, spricht Arendt die rechtliche Zwangslage von Flüchtlingen an und unterstreicht die Unmöglichkeit, auf das Recht zu verzichten, selbst wenn man dessen Grenzen erkennt: „For as much as the eternal insufficiency of law relegates man to the compassion of his fellow men, all the less can one demand of him that he replace the law with compassion." (Arendt 2007, 212) Anders als ein kritischer Ansatz, der Recht nur mit Herrschaft, Gewalt und Ausgrenzung identifiziert, betont Arendt die entscheidende Rolle, die juristische Fiktionen wie die der Rechtspersönlichkeit bei der fortschreitenden Gleichstellung der Menschen als eigenständige Individuen spielen können - ein immer unvollständiger und fragiler, aber keineswegs zu vernachlässigender Prozess. Ihre Analyse der Staatenlosigkeit führt sie zur Schlussfolgerung, dass Gleichheit nicht gegeben, inhärent oder natürlich ist; sie kann nur aus „the strength of our decision to guarantee ourselves mutually equal rights" (Arendt 1968, 301) entstehen. Diese Garantien finden innerhalb eines rechtlichen Rahmens eine gewisse relative Stabilität. Arendt findet im römischen Recht in dieser Hinsicht unerwartete Ressourcen, wie man an ihren Vermittlungsbemühungen, warum die juristische Person rechtlich und politisch von Bedeutung ist, sehen kann. Für Esposito ist die juristische Person bzw. die Maske des Rechts eine Form der Repräsentation, die das menschliche Gesicht verbirgt und entstellt und eine unmittelbare Begegnung mit dem singulären, konkreten, materiellen Lebewesen behindert. Für Arendt fungiert die Maske als eine Form der Selbstdarstellung, die es uns durch Verborgenheit erlaubt, auf der juristischen Bühne in Erwartung von Mitschauspieler*innen und Zu- 
schauer*innen aufzutreten, die unsere Gleichberechtigung als Rechtssubjekte bezeugen könnten. ${ }^{17}$

Arendts Diskussion von Staatenlosigkeit und Menschenrechten hebt die Bedeutung des Rechts für die fortschreitende Gleichstellung von Individuen hervor, ermutigt aber auch zu einer rigorosen Kritik an bestehenden Rechtsordnungen, indem mit der Notlage der von ihnen marginalisierten oder entrechteten Subjekte gerungen wird. In der Tat kann ihr eigentümliches Verständnis der juristischen Person als einer Maske, die das Gesicht bedeckt, aber auch die Stimme der Schauspielerin durchklingen lässt, als Brennpunkt für eine kritische Untersuchung des gegenwärtigen Menschenrechtsrahmens dienen: Inwieweit bietet dieser Rahmen verschiedenen Kategorien von Migrant*innen eine Rechtspersönlichkeit oder eine künstliche Maske, die ihr Antlitz verschleiert, damit sie als Rechtssubjekte erscheinen können und nicht als nacktes Leben, das willkürlicher staatlicher Gewalt ausgesetzt werden kann? Inwieweit lässt dieser Rahmen zu, dass die Rechtsansprüche von Migrant*innen „durchklingen“?

\section{Rechtspersönlichkeit, Territorialität und die zeitgenössischen Formen der Rechtlosigkeit}

Eine Arendt'sche Kritik der Menschenrechte nimmt eine neue Dringlichkeit an angesichts von Grenzkontrollpraktiken, die Migrant*innen effektiv „outside the pale of the law“ (Arendt 1968, 295) drängen und ihre Rechtspersönlichkeit untergraben. Besonders beunruhigend ist in diesem Zusammenhang die „Extraterritorialisierung“ oder „Externalisierung“ der Migrationskontrolle, die ein breites Spektrum von Praktiken umfasst, darunter bilaterale und multilaterale Vereinbarungen zur „Auslagerung“ oder „Ausfuhr“ der Grenzkontrolle in Herkunfts- und Transitländer, Seeoperationen, um Migrant*innen zu stoppen, bevor sie ihr Ziel erreichen können, Push-back-Operationen, die Migrant*innen zwangsweise in Staaten ausweisen, in denen sie ohne Rücksicht auf internationale Menschenrechtsnormen inhaftiert oder abgeschoben werden könnten, und der Ausschluss bestimmter Gebiete aus der Jurisdiktion, um Einwanderung zu verhindern (siehe z.B. Mitsilegas 2010). Die Inhaftierung haitianischer und kubanischer Asylsuchender durch die US-Regierung auf ihrem Marinestützpunkt in Guantánamo Bay in den

17 Diese Formulierung stützt sich auf Arendts Argumente über die phänomenale Natur der Welt; siehe Arendt 1978, 21-22. 
1980er und 1990er Jahren als Versuch, sie außerhalb der US-Jurisdiktion zu platzieren (siehe Dastyari 2007), und, jüngst, der Ausschluss des gesamten australischen Festlandes aus seiner Migrationszone, um automatisch alle „unbefugten Seeankömmlinge“ in Haftzentren auf Manus Island oder Nauru zu überstellen, sind in dieser Hinsicht zwei der berüchtigtsten Beispiele (siehe Vogl 2015). Die Extraterritorialisierung ist ein Versuch der „Dejuridifizierung“, um einen Begriff von Seyla Benhabib zu verwenden (Benhabib 2009, 702). Sie verfolgt das Ziel, sich den Verpflichtungen nach innerstaatlichem und internationalem Recht zu entziehen und den Migrant*innen jede rechtliche Stellung zu verweigern.

Inwieweit schaffen Menschenrechtsnormen heute Abhilfe gegen neue Manifestationen von Rechtlosigkeit, die sich aus der Extraterritorialisierung von Migrationskontrollen ergeben? Ein Fall des Europäischen Gerichtshofes für Menschenrechte (im Folgenden „der Gerichtshof“) aus dem Jahr 2012, Hirsi Jama and Others $v$. Italy, bietet einen nützlichen Brennpunkt, um die Hauptkonturen einer Arendt'schen Kritik der Menschenrechte als Antwort auf diese Frage neu zu überdenken. Die Antragsteller*innen in Hirsi waren elf somalische und dreizehn eritreische Staatsangehörige, die als Teil einer größeren Gruppe von etwa zweihundert Migrant*innen Libyen verließen, um nach Italien zu gelangen. Am 6. Mai 2009 fingen die italienische Polizei und Küstenwache ihre Schiffe in der Nähe von Lampedusa ab. Im Einklang mit der italienisch-libyschen Kooperation im Bereich der Migrationskontrolle wurden die Migrant*innen auf italienische Militärschiffe überstellt und nach Tripolis zurückgeschickt; Italien informierte die Migrant*innen nicht über ihr Ziel und unternahm keine Schritte, um sie zu identifizieren. ${ }^{18} \mathrm{Ihr}$ gesamter Besitz, einschließlich ihrer Identitätsdokumente, wurde vom italienischen Militärpersonal beschlagnahmt. Sobald die Migrant*innen in Tripolis angekommen waren, wurden sie den libyschen Behörden übergeben. Die italienische Operation war angesichts beunruhigender historischer Erinnerungen in Europa ziemlich schockierend; wie es in einem Bericht von Human Rights Watch lautet: „For the first time in the post-World War II era, a European state ordered its coast guard and naval vessels to interdict and forcibly return boat migrants on the high seas without doing any screening whatsoever to determine whether any passengers needed protection or were particularly vulnerable“ (Human Rights Watch 2009).

18 Zur italienischen Zusammenarbeit mit Libyen bei der Migrationskontrolle siehe Andrijasevic 2010, 150-154. 
Hirsi stellte gleich mehrere Menschenrechtsverletzungen in den Mittelpunkt: Die Antragsteller*innen behaupteten, dass Italien gegen Artikel 3 der Europäischen Menschenrechtskonvention (EMRK) verstoßen habe, der „Folter oder unmenschliche oder erniedrigende Behandlung oder Strafe" verbietet, und dass das Abfangen gegen das Non-Refoulement-Prinzip verstoßen habe, das durch Artikel 33 der Flüchtlingskonvention garantiert wird, welcher die Zurückweisung einer Person an einen Ort, an dem sie wahrscheinlich einer solchen Behandlung unterzogen wird, untersagt. Sie behaupteten auch, dass Italien durch die Rückführung nach Libyen ohne jeglichen Versuch, sie zu identifizieren oder ihre Fälle individuell zu beurteilen, gegen Artikel 4 des Protokolls Nr. 4 verstoßen habe, welcher die „Kollektivausweisung ausländischer Personen“ verbietet. Schließlich behaupteten die Antragsteller*innen, ihre Überstellung nach Libyen habe Artikel 13 der EMRK verletzt, da sie ihnen das Recht auf einen „wirksamen Rechtsbehelf“ vorenthalten habe. In seiner einstimmigen Entscheidung stimmte das Gericht den Antragsteller*innen zu und befand Italien in jedem Fall für schuldig, gegen die genannten Artikel verstoßen zu haben.

Das Hirsi-Urteil weist auf entscheidende Veränderungen hin, welche die Kodifizierung der Menschenrechtsnormen seit dem Ende des Zweiten Weltkriegs mit sich gebracht hat. Als Arendt den Zustand der Staatenlosigkeit als Zustand der Rechtlosigkeit beschrieb, verwies sie auf den Verlust der Rechtspersönlichkeit, der sich aus dem Fehlen jeglicher völkerrechtlicher Anerkennung ergab, insbesondere im Falle derjenigen, die de facto staatenlos waren (z.B. Flüchtlinge). Im Folgenden überdenke ich einige ihrer Schlüsselargumente in einer kritischen Analyse des Falls Hirsi. Es geht hier nicht darum, die entscheidende Bedeutung dieses Falles für die Menschenrechte von Migrant*innen außer Acht zu lassen; ich hoffe vielmehr, diese Dimensionen hervorzuheben, indem ich zeige, wie das Urteil des Gerichtshofs die Paradoxien der Menschenrechte neu verhandelt, um die Bemühungen der italienischen Regierung zurückzuweisen, diesen Migrant*innen die Rechtspersönlichkeit zu verweigern. Dieses Urteil unmissverständlich als Sieg zu lesen, hieße jedoch, die Art und Weise zu übersehen, wie es die Grenzen, Spannungen und Probleme in den bestehenden Formulierungen der Menschenrechte offenbart. Wenn ich kritisch über Hirsi nachdenke, dann betone ich die Schwächen des völkerrechtlichen Schutzes von Migrant*innen und verbinde dieses Problem mit der Tendenz, bei der Anwendung der Menschenrechte die territoriale Jurisdiktion und bei der Grenzkontrolle die Achtung vor dem souveränen Staat zur Norm zu erheben. Ausgehend von 
Arendts Betonung der Künstlichkeit und damit der Fragilität der juristischen Person schlage ich vor, die Rechtlosigkeit in Bezug auf den prekären Rechtsstatus von Migrant*innen neu zu überdenken, zumal diese Praktiken ausgesetzt sind, welche die Rechtspersönlichkeit im Leben und im Tod zunichtemachen.

Bei Hirsi $v$. Italy geht es um herausfordernde Fragen im Zusammenhang mit der Verfügbarkeit von Grundrechten für Migrant*innen, die auf hoher See abgefangen werden. Die italienische Regierung drängte das Gericht, den Fall für unzulässig zu erklären, indem sie Zweifel an der rechtlichen Stellung der Antragsteller*innen äußerte „who were not participating in the case in person“ (Hirsi v. Italy, §47). Sie machte geltend, dass den Vertreter*innen der Antragsteller*innen die erforderlichen Unterlagen zum Nachweis ihrer Vollmachten fehlten, dass sie den Kontakt zu einigen der Antragsteller*innen verloren hatten und dass die Identität der Antragsteller*innen nicht ordnungsgemäß festgestellt werden konnte. Tatsächlich versuchte der italienische Staat, diesen Migrant*innen die Rechtsstellung zu verweigern und sie zu Nichtpersonen zu machen, wobei er die Augen davor verschloss, dass es seine eigenen Grenzkontrollpraktiken waren, die zu Identifikationsproblemen führten und es den Antragsteller*innen unmöglich machten, vor Gericht anwesend zu sein.

Das Gericht wies diese problematische Position zurück und gewährte den Antragsteller*innen auf Basis der Unterschriften und Fingerabdrücke, die in der Akte zum Fall enthalten waren, Rechtsstellung. Zugleich machte es aber auch einen beunruhigenden Schritt, da es sich weigerte, seine Argumentation auf die beiden Antragsteller auszudehnen, die im Laufe des Verfahrens verstarben. Unter Berufung auf ihre bisherige Praxis, Fälle abzuweisen, „when an applicant dies during the course of the proceedings and no heir or close relative wishes to pursue the case“, beschloss es, „to strike the case out of the list in so far as it concerns Mohamed Abukar Mohamed and Hasan Shariff Abbirahman“ (Hirsi v. Italy, §57, §59). Aus rechtlicher Sicht verliert man mit dem Tod nicht notwendigerweise vollständig seine Rechtsstellung; die Toten können bestimmte Rechtsbeziehungen eingehen, insbesondere wenn es Beweise dafür gibt, dass ihre Zustimmung vor ihrem Tod eingeholt wurde (siehe Naffine 2009, 50). Was die Verweigerung der Rechtsstellung für die Verstorbenen in diesem Fall noch beunruhigender macht, ist die Wahrscheinlichkeit, dass die Handlungen der italienischen Regierung, insbesondere ihre Verletzung der Verbote des Non-Refoulement und der Kollektivausweisung, direkt oder indirekt zu deren Tod geführt haben könnten. Es wäre absurd vorzuschlagen, dass ein Staat mit solchen Aktionen un- 
gestraft davonkommen kann, solange es kein Opfer gibt, das noch am Leben ist, um auszusagen. In dieser Hinsicht unterscheidet sich der Fall der verstorbenen Antragsteller*innen nicht von denen, die „außerhalb des Schutzes des Gesetzes“ gestellt wurden, indem sie einem erzwungenen Verschwinden unterworfen wurden (siehe Artikel 2 des Internationalen Übereinkommens zum Schutz aller Personen vor dem Verschwindenlassen). In beiden Fällen ist es die staatliche Politik, welche die Menschen aus dem Bereich des Gesetzes hinausdrängt, es ihren Familien und Freunden erschwert, ihren Aufenthaltsort ausfindig zu machen, und ihr Leben zur Disposition stellt.

Die Verweigerung der Rechtsstellung für diese beiden verstorbenen Migranten beraubt sie ihrer Rechtspersönlichkeit oder, um an Arendts Argument zu erinnern, der künstlichen Maske der persona. Die Aktionen des italienischen Staates zielten darauf ab, diesen Migrant*innen jene Maske zu nehmen, um sie zu einem nackten Leben zu machen, das ungestraft willkürlicher Gewalt ausgesetzt werden kann. Die Entscheidung des Gerichtshofs versäumt es, diese Maske auch nach ihrem Tod wiederherzustellen, was auch bedeutet, dass ihre unverwechselbaren Stimmen nicht „durchklangen“, sodass ihre individuellen Lebensgeschichten und Schicksale nicht erzählt werden konnten. Tatsächlich erklärte das Gericht ohne eingehende Prüfung ihrer individuellen Fälle, dass ihre Ansprüche einfach „identisch“ mit jenen der anderen Antragsteller*innen seien (Hirsi v. Italy, §59). In dieser Hinsicht offenbart die Entscheidung die Grenzen der bestehenden Menschenrechtsnormen, insbesondere ihr Versagen, auf die Herausforderungen einer Einwanderungspolitik, welche Migrant*innen auf gefährlichere Routen drängt und ihr Todesrisiko erhöht, in vollem Umfang zu antworten.

Ebenso wichtig sind die Beschränkungen, die sich aus der Tendenz ergeben, bei der Anwendung von Menschenrechtsnormen die territoriale Jurisdiktion als Norm zu übernehmen (siehe Gammeltoft-Hansen 2011b, 107-108). Dieser Punkt verdient besondere Aufmerksamkeit, weil Hirsi als ein historisches Urteil, das die extraterritoriale Reichweite von Menschenrechtsnormen bekräftigt, begrüßt wurde. Mit den Worten von Sherif Elsayed-Ali, dem Leiter der Abteilung Rechte von Flüchtlingen und Migranten bei Amnesty International, stellt die Entscheidung fest, dass „states intercepting individuals outside their territorial waters cannot operate in a legal vacuum“ (Amnesty International 2012; siehe auch UNHCR 2012). Ein weitaus zweideutigeres Bild ergibt sich jedoch, wenn man sich vergegenwärtigt, wie das Urteil des Gerichtshofs die Territorialität als Norm bei der Behandlung von Fragen der Jurisdiktion, des Non-Refoulement und der Kollektivausweisung bekräftigt. 
In Bezug auf die Frage der Jurisdiktion versuchte Italien, sich jeder Verantwortung zu entziehen, indem es argumentierte, dass die fraglichen Ereignisse zwar auf italienischen Militärschiffen stattfanden, die italienischen Behörden jedoch keine „absolute and exclusive control“ über die Antragsteller*innen ausübten (Hirsi v. Italy, §64). Diese Schlussfolgerung beruhte auf einem streng territorialen Verständnis des Artikels 1 der EMRK, der die Vertragsstaaten verpflichtet, die in der Konvention artikulierten Rechte und Freiheiten für „everyone within their jurisdiction“ zu sichern. Es wertete das Abfangen als eine humanitäre Rettungsaktion und bestand darauf, dass die Operation keinen „link between the State and the persons concerned establishing the State’s jurisdiction“ (Hirsi v. Italy, §65) geschaffen habe. Der Gerichtshof stellte diese Ansicht infrage, indem er argumentierte, Italien habe nicht nur de jure die Jurisdiktion, da die Ereignisse auf Militärschiffen unter Flaggen stattfanden, sondern auch de facto die Jurisdiktion, da es „control and authority“ über die betreffenden Migrant*innen ausübte (Hirsi v. Italy, §74). Nach Ansicht mehrerer Kommentator*innen geht diese Einschätzung über eine streng territoriale Konzeption der Jurisdiktion hinaus, indem sie diese im Sinne einer Kontrolle über Personen neu überdenkt (siehe z. B. De Boer 2015; Giuffré 2012; Heijer 2013; Moreno-Lax 2012). Der Gerichtshof erkannte jedoch die italienische Jurisdiktion in diesem Fall an, ohne die Idee aufzugeben, dass die territoriale Jurisdiktion die Norm sei; tatsächlich betonte er immer wieder, dass „, $[\mathrm{t}]$ he jurisdiction of a State, within the meaning of Article 1, is essentially territorial“ und dass „[i]t is presumed to be exercised normally throughout the State’s territory“ (Hirsi v. Italy, §70 und §71; Hervorhebung hinzugefügt). Mit anderen Worten: Das Urteil ist keine Erklärung, welche die Reichweite der Menschenrechte auf alle Arten der extraterritorialen Einwanderungskontrolle pauschal bestätigt. Der Gerichtshof hob hervor, dass er eine Ausnahme von der Norm der territorialen Jurisdiktion mache - eine Ausnahme, über die je nach den Umständen jedes Einzelfalls entschieden werden müsse (Hirsi v. Italy, §73; siehe auch §72). Darüber hinaus ließ Hirsi die schwierigeren Fragen der Jurisdiktion unbeantwortet, die sich stellen, wenn die Migrationskontrolle mehrere, auch private Agent*innen einschließt sowie weniger offensichtliche Formen der Kontrolle über Personen (siehe Costello 2016, 246; Gammeltoft-Hansen 2011b, Kapitel 5; Klepp 2010; Mann 2013, 366-368).

In ähnlicher Weise manifestieren sich im Urteil des Gerichtshofs zur Frage des Refoulement auch die ungelösten Aporien im Bereich der Menschenrechte, insbesondere jene, die sich aus der langjährigen Praxis ergeben, 
in Fragen der Grenzkontrolle auf den souveränen Staat zurückzugreifen. Obwohl es in der EMRK keine spezifische Bestimmung gibt, die sich explizit auf das Asylrecht bezieht (siehe Mole und Meredith 2010, 19), hat der Gerichtshof die Frage des Non-Refoulement traditionell so behandelt, dass er Artikel 3 als Verbot der Ausweisung einer Person in ein Land gelesen hat, in dem sie einem „real risk“ ausgesetzt wäre, „torture or [...] inhuman or degrading treatment or punishment“ zu erleiden. Dementsprechend entschied er in der Rechtssache Hirsi, dass Italien die Antragsteller*innen nicht nur dem Risiko einer Misshandlung in Libyen, sondern auch dem Risiko einer willkürlichen Zurückweisung in ihre Herkunftsländer (d.h. Eritrea und Somalia) ausgesetzt habe. Während der Gerichtshof den Schutz des Non-Refoulement auf die fraglichen Migrant*innen ausdehnte, wertete er diesen Schutz jedoch auch als Ausnahme vom souveränen Recht „to control the entry, residence and expulsion of aliens“ (Hirsi v. Italy, §113). Die vom Gerichtshof angeführte Ausnahme hat zweideutige Effekte, da sie die Idee infrage stellt, dass das souveräne Recht, die Einreise zu kontrollieren, absolut sei. Allerdings tut sie dies, indem sie auch den problematischen Darstellungen Glauben schenkt, die von europäischen Staaten zur Rechtfertigung neuer Formen der Grenzkontrolle verwendet werden, wie man an den Hinweisen des Gerichtshofs auf „the burden and pressure“ erkennen kann, die „the increasing influx of migrants and asylum seekers“ auf Staaten „in the present context of economic crisis“ (Hirsi v. Italy, §122) ausübe.

Das Urteil des Gerichts wurde auch deswegen als beispiellos begrüßt, weil es das in Artikel 4 des Protokolls Nr. 4 der EMRK formulierte Verbot der „Kollektivausweisung von ausländischen Personen“ auf Migrant*innen ausdehnt, die sich nicht innerhalb der territorialen Grenzen eines Vertragsstaates aufhalten. Unter Betonung der Notwendigkeit, sich der EMRK als „a living instrument“ zu nähern, „which must be interpreted in the light of present-day conditions“, wies der Gerichtshof darauf hin, dass eine streng territoriale Auslegung der Kollektivausweisung, wie sie von der italienischen Regierung vertreten wird, Artikel 4 im Hinblick auf neue Formen der maritimen Migration „ineffective“ machen und Migrant*innen auf See ohne jeden Schutz zurücklassen würde. Auch in diesem Punkt unterstrich der Gerichtshof jedoch erneut, dass die Jurisdiktion im Wesentlichen territorial sei und dass „the notion of expulsion is also principally territorial“ (Hirsi v. Italy, $\S 178$; Hervorhebung hinzugefügt).

Kurz gesagt, das Urteil des Gerichtshofs in der Rechtssache Hirsi bekräftigt die territoriale Jurisdiktion als Norm und sieht eine extraterritoriale 
Anwendung der Menschenrechte als Ausnahme vor. Darüber hinaus wird die potenziell weitreichende Wirkung des Urteils, das von Kommentator*innen gepriesen wird, in vielerlei Hinsicht eingeschränkt, da der Gerichtshof die staatliche Souveränität respektiert und die neue Grenzkontrollpolitik als legitime Maßnahme anerkennt. ${ }^{19}$ Das Urteil verurteilt Italien, aber es tut dies, ohne das Recht des italienischen Staates, die Einreise in sein Hoheitsgebiet zu kontrollieren, infrage zu stellen; ganz im Sinne der Forderung der italienischen Regierung, der Gerichtshof solle „examine only the events of 6 May 2009 and not call into question Italy's powers as regards immigration control“, stellt das Urteil das souveräne Recht auf Grenzkontrolle nicht infrage und läuft sogar Gefahr, die einwanderungsfeindliche Rhetorik der meisten europäischen Regierungen zu übernehmen, die sich häufig über den „Zustrom“ von Migrant*innen beschweren, selbst wenn die tatsächlichen Zahlen diese Behauptung nicht stützen (Hirsi v. Italy, §100, §122). Das Urteil des Gerichts erkennt die Rechtspersönlichkeit der Migrant*innen an, die am 6. Mai 2009 abgefangen wurden (mit Ausnahme der beiden verstorbenen Antragsteller); aber wenn es um Millionen anderer Migrant*innen geht, die sich aufgrund von Visabeschränkungen oder anderen Grenzkontrollmaßnahmen nicht frei bewegen können, wird darin bestätigt, dass „personhood operates always [...] in the shadow of the border, and has often found itself stunted as a result“, um Linda Bosniaks Argument in einem anderen Kontext in Erinnerung zu rufen (Bosniak 2011, 210).

Das Hirsi-Urteil unterstreicht, dass Migrant*innen, insbesondere diejenigen, die abgefangen und zurückgedrängt werden, eine unsichere Rechtsstellung haben; sie haben Zugang zu einem viel beschränkteren Bündel von Rechten mit unsicheren Garantien. Mit anderen Worten, ihre Rechtspersönlichkeit ist prekär, was etymologisch „als Gefallen gegeben, abhängig von der Gunst eines anderen“ bedeutet und die Unsicherheit von Rechten unterstreicht, die von der Gunst und Diskretion einer anderen Person oder Behörde abhängen (siehe Fassin 2012, 4, 264-265Fn.; Gündoğdu 2015, 93). In dem Maße, wie menschenrechtliche Prozesse die territoriale Jurisdiktion im Zusammenhang mit internationaler Migration zur Norm machen, besteht die Gefahr, dass die Rechte abgefangener Migrant*innen zu Ausnahmen werden, über die im Einzelfall zu entscheiden ist. Das Hirsi-Urteil unterstreicht, dass das universelle Recht auf Rechtspersönlichkeit, das durch

19 Für eine Kritik der langjährigen Rücksichtnahme des Gerichtshofs auf den Staat, wenn es um Grenzkontrollen geht, siehe Dembour 2015. 
den bestehenden Menschenrechtsrahmen garantiert wird, nicht die von Arendt untersuchten „Aporien der Menschenrechte“ löst; die Kluft zwischen „Mensch“ und „Bürger“ wird in mancher Hinsicht überbrückt, indem man zum Konzept der „menschlichen Person“ übergeht, aber diese Überbrückung setzt uns einer Vielzahl von Grenzfiguren aus, die nun in einer Kluft zwischen „dem Menschen“ und „der Person“ leben.

Wenn man diese Probleme betrachtet, ist es ziemlich verlockend, sich Roberto Esposito anzuschließen, der argumentiert, dass Depersonalisierung oder das Zunichtemachen der Rechtspersönlichkeit dem dispositif der Person immanent sei und dass der Menschenrechtsrahmen, der die juristische Person als Grundlage nimmt, nicht anders kann, als neue Formen gewaltsamer Ausgrenzung hervorzubringen: „It isn't possible to personalize someone without depersonalizing or reifying others, without pushing someone over into the indefinite space that opens like a kind of trap door below the person.“ (Esposito 2012b, 24)

Der Arendt'sche Rahmen, der in diesem Kapitel angeboten wird, teilt mit Espositos Kritik die Idee, dass die universelle Rechtspersönlichkeit, welche durch die Menschenrechte garantiert wird, die „Aporien der Menschenrechte“ nicht auflöst, und er hebt auch hervor, wie Rechtspersönlichkeit gemacht und zunichtegemacht werden kann. Er widerspricht jedoch seiner Schlussfolgerung, dass Rechtspersönlichkeit einfach ein Mechanismus der Gewalt ist, der unaufhaltsam Ausschlüsse von und Hierarchien unter Menschen erzeugt. Stattdessen zielt der hier gewählte Rahmen darauf ab, das rechtliche Artefakt der juristischen Person in seinen zweideutigen Dimensionen zu verstehen, indem er sich mit seiner Anfälligkeit für Praktiken des Zunichtemachens ebenso befasst wie mit Möglichkeiten, die dieser für eine Gleichstellung unter den Menschen bieten kann. Darüber hinaus können aus Arendt'scher Sicht die Aporien im Bereich der Menschenrechte, einschließlich derer, die sich aus den Spannungen zwischen universeller Rechtspersönlichkeit und territorialer Souveränität ergeben, auf unterschiedliche Art und Weise umschifft werden, wobei einige davon vielversprechender sein können als andere, um die Rechtlosigkeit von Migrant*innen anzufechten. 


\section{Das Recht, Rechte zu haben: Rechtspersönlichkeit jenseits von Territorialität?}

Im Fall Hirsi liefert das gesonderte Gutachten des Richters Paulo Sérgio Pinto de Albuquerque einige entscheidende Erkenntnisse in dieser Hinsicht, da es die Grenzen der Argumentation des Gerichts aufzeigt und entscheidende Einsichten darüber bietet, wie wir die Rechtspersönlichkeit von Migrant*innen über die Grenzen der Territorialität hinaus neu denken können. ${ }^{20}$ Besonders interessant ist, dass Richter Pinto de Albuquerque seine Ausführungen mit einem Appell beginnt, der sich an Hannah Arendt anlehnt: „The ultimate question in this case is how Europe should recognise that refugees have ,the right to have rights. " (Hirsi v. Italy, 49) Ich schlage vor, dass diese gesonderte Stellungnahme Möglichkeiten eröffnet, einige der Beschränkungen bestehender Menschenrechtsnormen anzugehen und sie im Sinne eines „Rechts, Rechte zu haben“ neu zu überdenken, das die Anerkennung eines Rechts auf Rechtspersönlichkeit, politische Mitgliedschaft und Menschlichkeit fordert.

Besonders hervorzuheben ist in diesem Zusammenhang das weitergehende Verständnis des Non-Refoulement-Prinzips, das in der separaten Stellungnahme dargelegt wird. Während der Gerichtshof dieses Prinzip konventionell recht eng ausgelegt hat, um Migrant*innen vor ,torture or [...] inhuman or degrading treatment or punishment" zu schützen, schlägt Richter Pinto de Albuquerque gemäß Artikel 3 EMRK vor, dass ,the non-refoulement obligation can be triggered by a breach or the risk of a breach of the essence of any European Convention right" (Hirsi v. Italy, 50). Aufbauend auf der gängigen Idee, dass die Bestimmung des Flüchtlingsstatus lediglich deklaratorisch und nicht konstitutiv ist - d.h., man wird ein Flüchtling, wenn man die Kriterien der Flüchtlingskonvention von 1951 aufgrund faktischer Umstände erfüllt, auch wenn es keine formale Bestätigung dieses Status gibt (UNHCR 1977; siehe auch Hathaway 2005, 158-159; Hathaway und Foster 2014) -, dehnt die separate Stellungnahme den Schutz des Non-Refoulement potenziell auf jede migrantische Person unabhängig von ihrem Status aus: „As the determination of refugee status is merely declaratory, the princi-

20 Dies ist eine übereinstimmende Meinung, aber sie geht in vielerlei Hinsicht über die Argumentation und Schlussfolgerungen des Gerichtshofs hinaus, wie ich weiter unten erläutere. Für die Frage, warum Richter*innen separate Gutachten schreiben, wenn sie mit dem Urteil übereinstimmen, siehe White und Boussiakou 2009. 
ple of non-refoulement applies to those who have not yet had their status declared (asylum seekers) and even to those who have not expressed their wish to be protected." (Hirsi v. Italy, 51) Dieser Schritt ist vielversprechend im Hinblick auf die Destabilisierung der kategorischen Unterscheidungen, die selbst innerhalb der Menschenrechtsanwaltschaft und bei Prozessen regelmäßig zwischen den verschiedenen Kategorien von Migrant*innen getroffen werden, und eröffnet die Möglichkeit, Migrant*innen mit irregulärem Status in den Schutz des Non-Refoulement einzubeziehen.

Ein ähnlicher Ansatz, der das Prinzip der territorialen Souveränität infrage stellt, zeigt sich in den Ausführungen von Richter Pinto de Albuquerque zum Asylrecht. Die gesonderte Stellungnahme stellt das Territorialitätsprinzip der Flüchtlingskonvention infrage, welches besagt, dass man sich außerhalb seines Herkunftslandes aufhalten muss, um sich für Asyl zu qualifizieren, und schlägt vor, dass die Staaten eine positive Pflicht haben, in ihren Botschaften Menschen, die um Asyl ansuchen, Visa zu erteilen, und dass sie zur Verantwortung gezogen werden sollten, wenn sie dieser Pflicht nicht nachkommen (Hirsi v. Italy, 54). Diese Stellungnahme bezweifelt die Idee, dass Asyl lediglich ein souveränes Geschenk ist, und fordert uns auf, diese entscheidende Norm im Geiste von Arendts Vorschlag des „Rechts, Rechte zu haben" zu überdenken. Diese scharfen Worte, die den Staaten eine positive Pflicht auferlegen, unterscheiden sich deutlich von jenen im Urteil des Gerichtshofs, mit dem das souveräne Recht auf Grenzkontrolle in vielerlei Hinsicht bekräftigt wird. Sie ist auch anders und viel anspruchsvoller als die Sprache in der Allgemeinen Erklärung der Menschenrechte (AEMR), die ein „Recht, Asyl zu suchen und zu genießen“ anerkennt, aber nicht das Recht auf Asyl garantiert. Tatsächlich erwies sich diese Frage während der Ausarbeitung der AEMR als recht kontrovers; als mehrere Teilnehmer*innen vorschlugen, den Wortlaut in "the right to seek and to be granted asylum " zu ändern, um den Staaten eine positive Pflicht aufzuerlegen, lehnte die Mehrheit der Vertreter*innen der Staaten diesen Vorschlag mit der Begründung ab, dass er die Befugnisse souveräner Staaten in Bezug auf die Einwanderungskontrolle unangemessen einschränken würde..$^{21}$

Im Einklang mit ihrer stärkeren Ausformulierung des Asylrechts stellt die separate Stellungnahme auch die im Hirsi-Urteil vorgesehenen Reparationen infrage. Das Gericht forderte die italienische Regierung lediglich auf,

21 Für die Debatten während der Abfassung dieses Artikels der AEMR siehe Morsink 1999, 74-79. 
„to take all possible steps to obtain assurances from the Libyan authorities that the applicants will not be subjected to" Menschenrechtsverletzungen, einschließlich Refoulements (Hirsi v. Italy, §211). Doch dies ist bei Weitem kein zufriedenstellender Rechtsbehelf; schließlich stellte das Gericht selbst bei der Beurteilung der Behauptungen bezüglich der Verletzung von Artikel 3 (d.h. des Verbots von „Folter oder unmenschlicher oder erniedrigender Behandlung oder Strafe“) fest, dass die italienischen Behörden vernünftigerweise nicht hätten erwarten dürfen, dass Libyen „sufficient guarantees against arbitrary repatriation“ (Hirsi v. Italy, §152, siehe insbesondere $\S 153-156)$ bieten wird. Richter Pinto de Albuquerque hebt in seiner separaten Stellungnahme die Unzulänglichkeit dieses Rechtsmittels hervor und besteht darauf, dass die italienische Regierung auch eine positive Verpflichtung hat „to provide the applicants with practical and effective access to an asylum procedure in Italy" (Hirsi v. Italy, 59).

Wie die Verfasser*innen der AEMR erkannten, würde die stärkere Formulierung des Asylrechts ernsthafte Herausforderungen bei der Einwanderungskontrolle mit sich bringen, die bisher als ein Recht des souveränen Staates angesehen wurde. Tatsächlich bestätigte das Gericht im Fall Hirsi diese gängige Ansicht. Die gesonderte Stellungnahme fordert dagegen ein Überdenken der Menschenrechte im Sinne eines „Rechts, Rechte zu haben“, auch in seinem Beharren darauf, dass die Einwanderungskontrolle kein souveränes Recht ist, das gegen die Rechte von Migrant*innen ausgespielt werden darf, sondern stattdessen „a primary State function“, die im Lichte der Menschenrechtsnormen überprüft werden muss (Hirsi v. Italy, 57). Während der konventionelle Ansatz des Gerichtshofs den Migrant*innen die Last aufbürdet zu beweisen, dass ihre Fälle eine Ausnahme von der Norm der territorialen Souveränität verdienen, verschiebt die separate Stellungnahme die Last auf die Staaten, indem sie von ihnen verlangt sicherzustellen, dass ihre Grenzpolitik mit den Menschenrechtsnormen in Einklang steht. Darüber hinaus nimmt das Urteil des Gerichtshofs einen engen Fokus ein, da es sich der Extraterritorialisierung der Migrationskontrolle nähert, indem es den sehr spezifischen Fall der italienischen „Push-Back“-Operation im Jahr 2009 betrachtet und erklärt, dass diese eine Ausnahme von der Norm der territorialen Jurisdiktion darstelle. Die separate Stellungnahme hingegen stellt diese Operation in den breiteren Kontext dessen, was James Hathaway treffend als „politics of non-entrée“ bezeichnet, und weist auf verschiedene andere Formen der extraterritorialen Migrationskontrolle hin, die menschenrechtlichen Normen unterworfen werden sollten (Hathaway 1992; siehe auch Hat- 
haway 1992, 40-41; Gammeltoft-Hansen und Hathaway 2015). Dazu gehören die Visa-Entscheidungen in den Botschaften, die Pre-Clearance-Checks in den Häfen und Flughäfen fremder Länder oder die Bereitstellung von Mitteln zur Auslagerung der Migrationskontrolle in andere Länder (Hirsi v. Italy, 57). Man könnte hier auch das berüchtigte Abkommen zwischen der EU und der Türkei anführen, nach dem alle in Griechenland ankommenden Flüchtlinge automatisch in die Türkei zurückgeführt werden sollen. Solche Grenzkontrollpraktiken werden regelmäßig von Staaten durchgeführt und sie wurden als legitime Formen staatlichen Handelns gemäß dem Prinzip der territorialen Souveränität betrachtet. Aber dies sind auch die Politiken, die Migrant*innen zu Nichtpersonen im Leben und Tod machen, und es gibt keine Möglichkeit, die Menschenrechte im Sinne eines „Rechts, Rechte zu haben“ zu verstehen, ohne diese Politiken, die das Problem der Rechtlosigkeit heute noch verschärfen, zu hinterfragen.

Die separate Stellungnahme ebnet den Weg für eine solche Befragung, indem sie die Einwanderungskontrolle als eine Staatsfunktion definiert, die in Übereinstimmung mit Menschenrechtsnormen erfüllt werden muss, und so über die Grenzen des geltenden Völkerrechts hinausgeht (siehe auch Mann 2016, 170). Nehmen wir zum Beispiel das Argument von Richter Pinto de Albuquerque, dass Staaten für die in ihren Botschaften getroffenen Visa-Entscheidungen haftbar gemacht werden sollten. Die Verweigerung eines Visums wird im Allgemeinen nicht als Verstoß gegen das Non-Refoulement-Prinzip angesehen, auch wenn diese Entscheidung letztlich dazu führt, dass einem potenziellen Asylsuchenden der Zugang zu Schutz versperrt wird. Darüber hinaus begründet das Völkerrecht keine positive Pflicht der Staaten, ihre Visapolitik in Übereinstimmung mit dem Recht auf Asyl anzupassen. ${ }^{22}$ Dementsprechend erkennt selbst die UNHCR Visakontrollen als legitime Maßnahmen im Einklang mit dem souveränen Recht auf Einreisekontrolle an (Gammeltoft-Hansen 2011b, 134). Vielleicht aufgrund des Bewusstseins, dass das Völkerrecht, so wie es jetzt existiert und ausgelegt wird, nicht ausreicht, um Platz für ein Überdenken der Menschenrechte im Sinne des „Rechts, Rechte zu haben“, wie es Arendt vorschlägt, zu schaffen, beruft sich Richter Pinto de Albuquerque auf zwei historische Persönlichkeiten, die während des Zweiten Weltkriegs unter Missachtung bestehender Gesetze handelten und Staatenlosen Visa erteilten: Raoul Gustaf Wallenberg, Schwedens Sondergesandter in Budapest zwischen Juli und Dezember 
1944, und Aristides de Sousa Mendes, der portugiesische Generalkonsul von 1938 bis 1940 in Bordeaux, Frankreich. Die Bemühungen von Sousa Mendes, der sich im Jahr 1939 weigerte, den Bestimmungen im von den Nazis besetzten Frankreich zu folgen, mehr als 30.00o Menschen Visa verschaffte und schließlich seine diplomatische Laufbahn aufgeben musste, werden in der separaten Stellungnahme besonders hervorgehoben: „Had this episode taken place today, the Portuguese diplomat would have acted in full accordance with the standard of protection of the European Convention on $\mathrm{Hu}$ man Rights. Indeed, his action would have been the only acceptable response to those in need of international protection." (Hirsi v. Italy, 55)

Dies ist jedoch keineswegs eine selbstverständliche Schlussfolgerung und der Schutz, den die internationale Menschenrechtsgesetzgebung bietet, bleibt weiterhin hinter Arendts Begriff des „Rechts, Rechte zu haben“ zurück. Als Arendt dieses Recht als das „one right without which no other can materialize" (Arendt 1949, 37) vorschlug, argumentierte sie auch, dass es im „international law which still operate[d] in terms of reciprocal agreements and treaties between sovereign states“ (Arendt 1968, 298) keinen Platz dafür gäbe. Die Argumentation des Gerichtshofs im Fall Hirsi zeigt, dass trotz der vielen Entwicklungen auf dem Gebiet der Menschenrechte die Norm der territorialen Souveränität die Rechtspersönlichkeit von Migrant*innen weiterhin erheblich schmälert. Die gesonderte Stellungnahme von Richter Pinto de Albuquerque hebt jedoch hervor, dass die Aporien der Menschenrechte, insbesondere die Spannungen, die sich aus der Verflechtung von territorialer Souveränität und dem Recht auf Rechtspersönlichkeit ergeben, auf phantasievolle Weise so gesteuert werden können, dass sie die Möglichkeiten eröffnen, Migrant*innen vor dem Gesetz als Gleichberechtigte zu etablieren.

\section{Schlussfolgerung: Menschenrechte von den Rändern her befragen}

Der universelle Menschenrechtsdiskurs führt die „Rechtspersönlichkeit“ als Grundlage des Anspruchs auf Gleichberechtigung ein. Damit will er die Lücke zwischen „Mensch“ und „Bürger*in“ schließen, welche die Rechtserklärungen des 18. Jahrhunderts geprägt hat. Es ist durchaus verlockend zu glauben, dass der Übergang von der Staatsbürgerschaft zur Rechtspersönlichkeit das grundlegende Problem der Rechtlosigkeit löst, das Arendt in ihrer Diskussion der Staatenlosigkeit untersucht hat. Schließlich war eines der bestimmenden Merkmale dieses Zustands der Verlust der Rechtspersönlichkeit. In einem nationalstaatlichen Rahmen, der die Rechtsstellung an 
die Staatsbürgerschaft knüpfte, wurden diejenigen, die staatenlos wurden, faktisch zu Nichtpersonen, die zur Rechtlosigkeit verbannt wurden. Dieses Problem kann, so könnte man folgern, nicht mehr bestehen, sobald jedem Menschen unabhängig vom Status der Staatsbürgerschaft Rechtspersönlichkeit zugeschrieben wird.

Was sich jedoch aus der oben angebotenen kritischen Analyse ergibt, ist ein viel zweideutigeres Bild dieser neuartigen Entwicklung. Die Entstehung eines universellen Menschenrechtsdiskurses, der jedem Menschen das Recht zugesteht, vor dem Gesetz als Person anerkannt zu werden, ist insofern eine willkommene Entwicklung, als sie es Migrant*innen ermöglicht, Rechtsstellung zu erhalten und Rechte geltend zu machen. Wie Arendts Bemerkungen zur Rechtspersönlichkeit unterstreichen, ist eine solche formale Anerkennung nicht nur eine juristische Angelegenheit; die künstliche Maske der juristischen Person ist untrennbar mit der Anerkennung der eigenen Stellung innerhalb einer politischen Gemeinschaft und der Menschheit verbunden. Arendts Darstellung der Rechtspersönlichkeit bringt jedoch auch die Zerbrechlichkeit dieser Maske ans Licht und fordert uns dazu auf, die verschiedenen Praktiken zu untersuchen, mit denen diese Maske zunichtegemacht wird. Die rechtliche Stellung von Migrant*innen ist durch verschiedene Grenzkontrollpolitiken, die darauf abzielen, Zonen der Gesetzlosigkeit zu schaffen, prekär geworden. Wie der Fall Hirsi unterstreicht, offenbart diese Prekarität die anhaltenden Aporien der Menschenrechte, einschließlich der Spannungen, die sich aus der Artikulation einer gleichberechtigten Rechtspersönlichkeit in einem System ergeben, das auf dem Prinzip der territorialen Souveränität beruht.

Als Arendt vorschlug, dass „ein Recht, Rechte zu haben“ nur von der Menschheit selbst garantiert werden könne, fügte sie auch hinzu, ,it is by no means certain whether this is possible" (Arendt 1968, 298). Wenn wir auf den Friedhof des Mittelmeers blicken, ist es unmöglich, diese Ungewissheit heute nicht zu teilen. Es ist genau diese Ungewissheit, die eine radikale Infragestellung der Menschenrechte erfordert, eine Infragestellung, die sich auf die liminalen Gestalten konzentriert, die an den Grenzen dieser Rechte angesiedelt sind. Diese Kritik sollte danach streben, nicht nur die Einschränkungen und Ausschlüsse der Menschenrechte zu verstehen, sondern auch die Möglichkeiten, die diese für die fortschreitende Gleichstellung der Menschen bieten könnten. 


\section{Literatur}

Agamben, Giorgio. 1998. Homo Sacer: Sovereign Power and Bare Life. Übersetzt von Daniel Heller-Roazen. Stanford, CA: University of Stanford Press.

Amnesty International. 2012. Italy: 'Historic' European Court Judgment Upholds Migrants' Rights. http://www.amnesty.org/en/news/italy-historic-european-court-judgment-upholds-migrants-rights-2012-02-23 [zuletzt aufgerufen am 26.6.2017].

Andrijasevic, Rutvica. 2010. „From Exception to Excess: Detention and Deportation across the Mediterranean Space“. In The Deportation Regime: Sovereignty, Space, and the Freedom of Movement, herausgegeben von Nicholas De Genova und Nathalie Peutz, 147-165. Durham, NC: Duke University Press.

Arendt, Hannah. 1949. „The Rights of Man’: What Are They?“ Modern Review 3 (1): 25-37.

Arendt, Hannah. 1968 (1951). The Origins of Totalitarianism. San Diego: Harcourt Brace Jovanovich.

Arendt, Hannah. 1998 (1958). The Human Condition. Chicago: University of Chicago Press.

Arendt, Hannah. 1990 (1963). On Revolution. London und New York: Penguin.

Arendt, Hannah. 1978. The Life of the Mind, Bd. 1. New York: Harcourt Brace Jovanovich.

Arendt, Hannah. 2003a. „Prologue“. In Responsibility and Judgment, herausgegeben von Jerome Kohn, 3-14. New York: Schocken Books.

Arendt, Hannah. 2003b. Denktagebuch, 1950 bis 1973, Bd. 1. Herausgegeben von Ursula Ludz und Ingeborg Nordmann. München: Piper.

Arendt, Hannah. 2005. „Introduction into Politics“. In The Promise of Politics, herausgegeben von Jerome Kohn, 93-200. New York: Schocken Books.

Arendt, Hannah. 2007. „Guests from No-Man's-Land“. In The Jewish Writings, herausgegeben von Jerome Kohn und Ron H. Feldman, 211-213. New York: Schocken Books.

Balibar, Étienne. 2002. Politics and the Other Scene. Übersetzt von Christine Jones, James Swenson und Chris Turner. London und New York: Verso.

Belting, Hans. 2017. Face and Mask: A Double History. Übersetzt von Thomas S. Hansen and Abby J. Hansen. Princeton und Oxford: Princeton University Press.

Benhabib, Seyla. 2004. The Rights of Others: Aliens, Residents and Citizens. Cambridge und New York: Cambridge University Press.

Benhabib, Seyla. 2009. „Claiming Rights across Borders: International Human Rights and Democratic Sovereignty“. American Political Science Review 103 (4): 691-704.

Bosniak, Linda. 2010. „Persons and Citizens in Constitutional Thought“. International Journal of Constitutional Law 8 (1): 9-29. 
Bosniak, Linda. 2011. „Human Rights within One State: Dilemmas of Personhood in Liberal Constitutional Thought“. In Are Human Rights for Migrants? Critical Reflections on the Status of Irregular Migrants in Europe and the United States, herausgegeben von Marie-Bénédicte Dembour und Tobias Kelly, 201-221. Abingdon, Oxon und New York: Routledge.

Boswell, Christina. 2003. „The 'External Dimension' of EU Immigration and Asylum Policy“. International Affairs 79 (3): 619-638.

Butler, Judith. 2004. Precarious Life: The Powers of Mourning and Violence. London und New York: Verso.

Cacho, Lisa Marie. 2012. Social Death: Racialized Rightlessness and the Criminalization of the Unprotected. New York und London: New York University Press.

Cohen, Jean. 1999. „Changing Paradigms of Citizenship and the Exclusiveness of the Demos“. International Sociology 14 (3): 245-268.

Costello, Cathryn. 2016. The Human Rights of Migrants and Refugees in European Law. Oxford: Oxford University Press.

Dastyari, Azadeh. 2007. „Refugees on Guantanamo Bay: A Blue Print for Australia’s 'Pacific Solution'?". AQ: Australian Quarterly 79 (1): 4-8, 40.

Dayan, Colin. 2011. The Law is a White Dog: How Legal Rituals Make and Unmake Personhood. Princeton, NJ: Princeton University Press, 2011.

De Boer, Tom. 2015. „Closing Legal Black Holes: The Role of Extraterritorial Jurisdiction in Refugee Rights Protection“. Journal of Refugee Studies 28 (1): 118-134.

Dembour, Marie-Bénédicte. 2012. „Hirsi (Part II): Another Side to the Judgment“. Strasbourg Observers, March 2. http://strasbourgobservers.com/2012/03/02/ hirsi-part-ii-another-side-to-the-judgment/ [zuletzt aufgerufen am 19.6.2017].

Dembour, Marie-Bénédicte. 2015. When Humans Become Migrants: Study of the European Court of Human Rights with an Inter-American Counterpoint. Oxford, UK: Oxford University Press.

Esposito, Roberto. 2012a. Third Person: Politics of Life and Philosophy of the Impersonal. Übersetzt von Zakiya Hanafi. Cambridge: Polity.

Esposito, Roberto. 2012b. „The Dispositif of the Person“. Law, Culture and the Humanities 8 (1): 17-30.

Esposito, Roberto. 2015. Persons and Things: From the Body's Point of View. Übersetzt von Zakiya Hanafi. Cambridge: Polity.

Fassin, Didier. 2012. Humanitarian Reason: A Moral History of the Present. Berkeley und Los Angeles, California: University of California Press.

Frontex. 2016. „Risk Analysis for 2016“. http://frontex.europa.eu/assets/Publications/Risk_Analysis/Annula_Risk_Analysis_2016.pdf [zuletzt aufgerufen am 30.6.2017]. 
Gammeltoft-Hansen, Thomas. 2011a. „Outsourcing Asylum: The Advent of Protection Lite“. In Europe in the World: EU Geopolitics and the Making of European Space, herausgegeben von Luiza Bialasiewicz, 129-152. Burlington, VT: Ashgate.

Gammeltoft-Hansen, Thomas. 2011b. Access to Asylum: International Refugee Law and the Globalisation of Migration Control. Cambridge: Cambridge University Press.

Gammeltoft-Hansen, Thomas, und James C. Hathaway. 2015. „Non-Refoulement in a World of Cooperative Deterrence“. Columbia Journal of Transnational Law 53 (2): $235-284$.

Giuffré, Mariagiulia. 2012. „Watered-Down Rights on the High Seas: Hirsi Jamma and Others $v$. Italy". International and Comparative Law Quarterly 61 (3): 728750.

Goldoni, Marco, und Christopher McCorkindale, Hrsg. 2012. Hannah Arendt and the Law. Oxford: Hart.

Goodwin-Gill, Guy S. 1996. The Refugee in International Law, Oxford und New York: Oxford University Press.

Grant, Stefanie. 2011. „Irregular Migration and Frontier Deaths: Acknowledging a Right to Identity“. In Are Human Rights for Migrants? Critical Reflections on the Status of Irregular Migrants in Europe and the United States, herausgegeben von Marie-Bénédicte Dembour und Tobias Kelly, 48-70. Abingdon, Oxon und New York: Routledge.

Gündoğdu, Ayten. 2015. Rightlessness in an Age of Rights: Hannah Arendt and the Contemporary Struggles of Migrants. Oxford und New York: Oxford University Press.

Haddad, Emma. 2008. „The External Dimension of EU Refugee Policy: A New Approach to Asylum“. Government and Opposition 43 (2): 190-205.

Hathaway, James C. 1992. „The Emerging Politics of Non-Entrée“. Refugees 91: 40-41.

Hathaway, James C. 2005. The Rights of Refugees under International Law. Cambridge und New York: Cambridge University Press.

Hathaway, James C., und Michelle Foster. 2014. The Law of Refugee Status, Cambridge: Cambridge University Press.

Heijer, Maarten den. 2013. „Reflections on Refoulement and Collective Expulsion in the Hirsi Case“. International Journal of Refugee Law 25 (2): 265-290.

Hirsi Jamaa and Others v. Italy. 2012. Application No. 27765/o9. Council of Europe: European Court of Human Rights, February 23, 2012. http://hudoc.echr. coe.int/eng?i=001-109231 [zuletzt aufgerufen am 26.9.2016].

Human Rights Watch. 2009. Pushed Back, Pushed Around: Italy's Forced Return of Boat Migrants and Asylum Seekers, Libya's Mistreatment of Migrants and Asylum Seekers. https://www.hrw.org/report/2009/09/21/pushed-back-pushedaround/italys-forced-return-boat-migrants-and-asylum-seekers [zuletzt aufgerufen am 4.7.2017]. 
Hyndman, Jennifer, und Alison Mountz. 2008. „Another Brick in the Wall? Neo-Refoulement and the Externalization of Asylum by Australia and Europe“. Government and Opposition 43 (2): 249-269.

Jacobson, David. 1996. Rights Across Borders: Immigration and the Decline of Citizenship. Baltimore: Johns Hopkins University Press.

Kalpouzos, Ioannis, und Itamar Mann. 2017. „The Banality of Crimes against Migrants“. Spiegel Online, 28. Oktober. http://www.spiegel.de/international/world/editorial-on-crimes-against-migrants-a-1175239.html [zuletzt aufgerufen am 14.11.2017].

Klepp, Silja. 2010. „A Contested Asylum System: The European Union between Refugee Protection and Border Control in the Mediterranean Sea“. European Journal of Migration and Law 12 (1): 1-21.

Mann, Itamar. 2013. „Dialectic of Transnationalism: Unauthorized Migration and Human Rights, 1993-2013“. Harvard International Law Journal 54 (2): 315-391.

Mann, Itamar. 2016. Humanity at Sea: Maritime Migration and the Foundations of International Law. New York: Cambridge University Press.

Mann, Itamar. Im Erscheinen. „Maritime Legal Black Holes: Migration and Rightlessness in International Law“. European Journal of International Law.

Maxwell, Lida. 2012. „Toward an Agonistic Understanding of Law: Law and Politics in Hannah Arendt's Eichmann in Jerusalem". Contemporary Political Theory 11 (1): 88-108.

Mezzadra, Sandro, und Brett Neilson. 2013. Border as Method, or, the Multiplication of Labor. Durham und London: Duke University Press.

Mitsilegas, Valsamis. 2010. „Extraterritorial Immigration Control in the 21st Century: The Individual and the State“. In Extraterritorial Immigration Control: Legal Challenges, herausgegeben von Bernard Ryan und Valsamis Mitsilegas, 39-65. Leiden und Boston: Martinus Nijhoff Publishers.

Mole, Nuala, und Catherine Meredith. 2010. Asylum and the European Convention on Human Rights. Council of Europe Publishing, Human Rights Files, No. 9. www.unhcr.org/refworld/pdfid/4eegbo972.pdf [zuletzt aufgerufen am 6.7.2017].

Moreno-Lax, Violeta. 2012. „Hirsi Jamaa and Others v Italy or the Strasbourg Court versus Extraterritorial Migration Control?“. Human Rights Law Review 12 (3): 574-598.

Morgades, Sílvia. 2008. „The Externalisation of the Asylum Function in the European Union“. In The Refugee in International Society: Between Sovereigns, herausgegeben von Emma Haddad, 161-185. Cambridge: Cambridge University Press, 2008.

Morsink, Johannes. 1999. The Universal Declaration of Human Rights: Origins, Drafting, and Intent. Philadelphia, Pennsylvania: University of Pennsylvania Press. 
Naffine, Ngaire. 2009. Law's Meaning of Life: Philosophy, Darwin and the Legal Person. Oxford und Portland, OR: Hart, 2009.

Nail, Thomas. 2016. Theory of the Border. New York: Oxford University Press.

Parekh, Serena. 2017. Refugees and the Ethics of Forced Displacement. New York: Routledge.

Pettit, Philip. 1997. Republicanism: A Theory of Freedom and Government. Oxford und New York: Oxford University Press.

Rajaram, Prem Kumar, und Carl Grundy-Warr. 2004. „The Irregular Migrant as Homo Sacer: Migration and Detention in Australia, Malaysia, and Thailand“. International Migration 42 (1): 33-64.

Rancière, Jacques. 1999. Disagreement: Politics and Philosophy. Übersetzt von Julie Rose. Minneapolis: University of Minnesota Press.

Robins, Simon, Iosif Kovras und Anna Vallianatou. 2014. „Addressing Migrant Bodies on Europe's Southern Frontier: An Agenda for Research and Practice“. Policy Brief, Centre for Applied Human Rights, University of York.

Rygiel, Kim. 2014. „In Life Through Death: Transgressive Citizenship at the Border“. In Routledge Handbook of Global Citizenship Studies, herausgegeben von Engin F. Isin und Peter Nyers, 62-72. London und New York: Routledge.

Sassen, Saskia. 2002. „The Repositioning of Citizenship: Emergent Subjects and Spaces for Politics“. Berkeley Journal of Sociology 46: 4-26.

Soysal, Yasemin Nuhoğlu. 1994. Limits of Citizenship: Migrants and Postnational Membership in Europe. Chicago: University of Chicago.

Trendelenburg, Adolf. 1910. A Contribution to the History of the Word Person. Übersetzt von Carl H. Haessler. Chicago: The Open Court Publishing.

UN General Assembly (UN-Generalversammlung). 2017. „Unlawful Death of Refugees and Migrants". A/72/335, 15. August. http://www.refworld.org/docid/59b923524.html [zuletzt aufgerufen am 14.11.2017].

UNHCR (United Nations High Commissioner for Refugees). 1977. „Note on Determination of Refugee Status under International Instruments“. EC/SCP/5, 24. August. http://www.unhcr.org/en-us/excom/scip/3ae68cco4/note-determination-refugee-status-under-international-instruments.html [zuletzt aufgerufen am 29.6.2017].

UNHCR. 2012. „Landmark Judgment of the Strasbourg Court on Push-Backs in the Mediterranean Sea“. http://www.unhcr.org/4f4619f76.html [zuletzt aufgerufen am 26.6.2017].

Vaughan-Williams, Nick. 2009. Border Politics: The Limits of Sovereign Power. Edinburgh: Edinburgh University Press.

Vogl, Anthea. 2015. „Over the Borderline: A Critical Inquiry into the Geography of Territorial Excision and the Securitisation of the Australian Border". University of New South Wales Law Journal 38 (1): 114-145. 
Volk, Christian. 2015. Arendtian Constitutionalism: Law, Politics, and the Order of Freedom. Oxford: Hart Publishing.

Waldron, Jeremy. 2000. „Arendt's Constitutional Politics“. In The Cambridge Companion to Hannah Arendt, herausgegeben von Dana Villa, 201-219. Cambridge und New York: Cambridge University Press.

White, Robin C. A., und Iris Boussiakou. 2009. „Separate Opinions in the European Court of Human Rights“. Human Rights Law Review 9 (1): 37-6o. 
